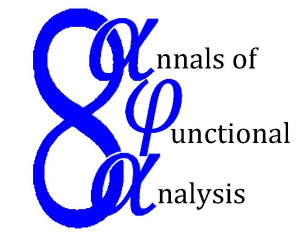

Ann. Funct. Anal. 5 (2014), no. 1, 77-93

$\mathscr{A}$ NNALS OF $\mathscr{F}$ UNCTIONAL $\mathscr{A}$ NALYSIS

ISSN: 2008-8752 (electronic)

URL:www.emis.de/journals/AFA/

\title{
NEW GRÜSS TYPE INEQUALITIES FOR RIEMANN-STIELTJES INTEGRAL WITH MONOTONIC INTEGRATORS AND APPLICATIONS
}

\author{
MOHAMMAD W. ALOMARI ${ }^{1 *}$ AND SEVER S. DRAGOMIR ${ }^{2}$ \\ Dedicated to Professor Tsuyoshi Ando with affection
}

Communicated by T. Riedel

\begin{abstract}
In this paper several new inequalities of Grüss' type for RiemannStieltjes integral with monotonic nondecreasing integrators under various assumptions for integrands are proved. Applications for functions of selfadjoint operators on complex Hilbert spaces are provided as well.
\end{abstract}

\section{INTRODUCTION}

The Čebyšev functional defined by

$$
\mathcal{T}(f, g)=\frac{1}{b-a} \int_{a}^{b} f(t) g(t) d t-\frac{1}{b-a} \int_{a}^{b} f(t) d t \cdot \frac{1}{b-a} \int_{a}^{b} g(t) d t
$$

has interesting applications in the approximation of the integral of a product as pointed out in the references below.

The problem of bounding the Čebyšev functional has a long history, starting with Grüss [21] inequality in 1935, where he had proved that for two integrable functions $f, g$ such that $\phi \leq f(x) \leq \Phi$ and $\gamma \leq f(x) \leq \Gamma$ for any $x \in[a, b]$, the inequality

$$
|C(f, g)| \leq \frac{1}{4}(\Phi-\phi)(\Gamma-\gamma)
$$

holds, and the constant $\frac{1}{4}$ is the best possible.

Date: Received: 25 June 2013; Accepted: 22 September 2013.

* Corresponding author.

2000 Mathematics Subject Classification. Primary 26D10; Secondary 26D15, 47A63.

Key words and phrases. Grüss inequality, Functions of bounded variation, Hölder continuous functions, Riemann-Stieltjes integral, Selfadjoint operators, Functions of selfadjoint operators. 
After that many authors have studied the functional (1.1) and therefore, several bounds under various assumptions for the functions involved have been obtained. For some new results and generalizations the reader may refer to [1]-[20], [23] and the references therein.

One of the recent generalization of (1.1) was considered by Dragomir in [9]. Namely, he has introduced the following Čbyšev functional for the RiemannStieltjes integral

$$
\begin{aligned}
\mathcal{T}(f, g ; u) & :=\frac{1}{u(b)-u(a)} \int_{a}^{b} f(t) g(t) d u(t) \\
& -\frac{1}{u(b)-u(a)} \int_{a}^{b} f(t) d u(t) \cdot \frac{1}{u(b)-u(a)} \int_{a}^{b} g(t) d u(t)
\end{aligned}
$$

under the assumptions that $f, g$ are continuous on $[a, b]$ and $u$ is of bounded variation on $[a, b]$ with $u(b) \neq u(a)$.

By simple computations with the Riemann-Stieltjes integral, Dragomir [9] established the identity:

$$
\begin{aligned}
\mathcal{T}(f, g ; u) & =\frac{1}{u(b)-u(a)} \int_{a}^{b}\left[f(t)-\frac{f(a)+f(b)}{2}\right] \\
& \cdot\left[g(t)-\frac{1}{u(b)-u(a)} \int_{a}^{b} g(s) d u(s)\right] d u(t),
\end{aligned}
$$

to obtain several sharp bounds of the Čebyšev functional for the RiemannStieltjes integral (1.3).

In this paper, some new Grüss' type inequalities for the Riemann-Stieltjes integral with monotonic nondecreasing integrators are proved. Applications for functions of selfadjoint operators on complex Hilbert spaces via the spectral representation theorem are provided as well.

\section{The Results}

We may start with the following result:

Theorem 2.1. Let $f:[a, b] \rightarrow \mathbb{C}$ be a $p-H_{f}-$ Hölder continuous function on $[a, b]$, where $p \in(0,1]$ and $H_{f}>0$ are given. Let $g, u:[a, b] \rightarrow \mathbb{R}$ be such that $g$ is Riemann-Stieltjes integrable with respect to a monotonic non-decreasing function $u$ on $[a, b]$ and there exists the real numbers $\gamma, \Gamma$ such that $\gamma \leq g(x) \leq \Gamma$ for all $x \in[a, b]$, then

$$
|\mathcal{T}(f, g ; u)| \leq \frac{1}{2^{p+1}} H_{f}(\Gamma-\gamma)(b-a)^{p}
$$


Proof. Taking the modulus in (1.4) and utilizing the triangle inequality, we get

$$
\begin{aligned}
|\mathcal{T}(f, g ; u)| & \leq \frac{1}{u(b)-u(a)} \int_{a}^{b}\left|f(t)-\frac{f(a)+f(b)}{2}\right| \\
& \times\left|g(t)-\frac{1}{u(b)-u(a)} \int_{a}^{b} g(s) d u(s)\right| d u(t) \\
& \leq \frac{1}{u(b)-u(a)} \sup _{t \in[a, b]}\left|f(t)-\frac{f(a)+f(b)}{2}\right| \\
& \times \int_{a}^{b}\left|g(t)-\frac{1}{u(b)-u(a)} \int_{a}^{b} g(s) d u(s)\right| d u(t) .
\end{aligned}
$$

Now, using the same approach considered in [11], we define

$$
I(g):=\frac{1}{u(b)-u(a)} \int_{a}^{b}\left(g(t)-\frac{1}{u(b)-u(a)} \int_{a}^{b} g(s) d u(s)\right)^{2} d u(t) .
$$

Then, we have

$$
\begin{aligned}
I(g) & =\frac{1}{u(b)-u(a)} \int_{a}^{b}\left[g^{2}(t)-2 g(t) \frac{1}{u(b)-u(a)} \int_{a}^{b} g(s) d u(s)\right. \\
& \left.+\left(\frac{1}{u(b)-u(a)} \int_{a}^{b} g(s) d u(s)\right)^{2}\right] d u(t) \\
& =\frac{1}{u(b)-u(a)} \int_{a}^{b} g^{2}(t) d u(t)-\left(\frac{1}{u(b)-u(a)} \int_{a}^{b} g(s) d u(s)\right)^{2}
\end{aligned}
$$

and

$$
\begin{aligned}
I(g) & =\left(\Gamma-\frac{1}{u(b)-u(a)} \int_{a}^{b} g(s) d u(s)\right)\left(\frac{1}{u(b)-u(a)} \int_{a}^{b} g(s) d u(s)-\gamma\right) \\
& -\frac{1}{u(b)-u(a)} \int_{a}^{b}(\Gamma-g(s))(g(s)-\gamma) d u(s) .
\end{aligned}
$$

As $\gamma \leq g(t) \leq \Gamma$, for all $t \in[a, b]$, then

$$
\int_{a}^{b}(\Gamma-g(s))(g(s)-\gamma) d u(s) \geq 0
$$

which implies

$$
\begin{aligned}
& I(g) \\
& \leq\left(\Gamma-\frac{1}{u(b)-u(a)} \int_{a}^{b} g(s) d u(s)\right)\left(\frac{1}{u(b)-u(a)} \int_{a}^{b} g(s) d u(s)-\gamma\right) \\
& \leq \frac{1}{4}(\Gamma-\gamma)^{2} .
\end{aligned}
$$

Using Cauchy-Buniakowski-Schwarz's integral inequality we have

$$
I(g) \geq\left[\frac{1}{u(b)-u(a)} \int_{a}^{b}\left|g(t)-\frac{1}{u(b)-u(a)} \int_{a}^{b} g(s) d u(s)\right| d u(t)\right]^{2}
$$


and thus by (2.3) we get

$$
\frac{1}{u(b)-u(a)} \int_{a}^{b}\left|g(t)-\frac{1}{u(b)-u(a)} \int_{a}^{b} g(s) d u(s)\right| d u(t) \leq \frac{1}{2}(\Gamma-\gamma) \text {. }
$$

Now, since $f$ is of $p-H_{f}$-Hölder type on $[a, b]$, then we have

$$
\begin{aligned}
\left|f(t)-\frac{f(a)+f(b)}{2}\right| & =\left|\frac{f(t)-f(a)+f(t)-f(b)}{2}\right| \\
& \leq \frac{1}{2}|f(t)-f(a)|+\frac{1}{2}|f(t)-f(b)| \\
& \leq \frac{H_{f}}{2}\left[(t-a)^{p}+(b-t)^{p}\right] .
\end{aligned}
$$

It follows that:

$$
\sup _{t \in[a, b]}\left|f(t)-\frac{f(a)+f(b)}{2}\right| \leq H_{f}\left(\frac{b-a}{2}\right)^{p} .
$$

Combining (2.4) and (2.5) with (2.2), we get

$$
\begin{aligned}
|\mathcal{T}(f, g ; u)| & \leq \frac{1}{u(b)-u(a)} \sup _{t \in[a, b]}\left|f(t)-\frac{f(a)+f(b)}{2}\right| \\
& \times \int_{a}^{b}\left|g(t)-\frac{1}{u(b)-u(a)} \int_{a}^{b} g(s) d u(s)\right| d u(t) \\
& \leq \frac{H_{f}}{2^{p+1}}(\Gamma-\gamma)(b-a)^{p}
\end{aligned}
$$

as required.

Corollary 2.2. Let $g, u$ be as in Theorem 2.1. If $f:[a, b] \rightarrow \mathbb{C}$ is $L_{f}$-Lipschitz on $[a, b]$, then

$$
|\mathcal{T}(f, g ; u)| \leq \frac{1}{4} L_{f}(\Gamma-\gamma)(b-a)
$$

When the function $f$ is of bounded variation we can state the following result as well:

Theorem 2.3. Let $g, u$ be as in Theorem 2.1. Let $f:[a, b] \rightarrow \mathbb{C}$ be a function of bounded variation on $[a, b]$, then we have

$$
|\mathcal{T}(f, g ; u)| \leq \frac{1}{4}(\Gamma-\gamma) \bigvee_{a}^{b}(f)
$$

The constant $\frac{1}{4}$ is best possible.

Proof. As in Theorem 2.1, we observe that

$$
\begin{aligned}
& |\mathcal{T}(f, g ; u)| \\
& \leq \frac{1}{u(b)-u(a)} \sup _{t \in[a, b]}\left|f(t)-\frac{f(a)+f(b)}{2}\right| \\
& \times \int_{a}^{b}\left|g(t)-\frac{1}{u(b)-u(a)} \int_{a}^{b} g(s) d u(s)\right| d u(t),
\end{aligned}
$$


and

$$
\frac{1}{u(b)-u(a)} \int_{a}^{b}\left|g(t)-\frac{1}{u(b)-u(a)} \int_{a}^{b} g(s) d u(s)\right| d u(t) \leq \frac{1}{2}(\Gamma-\gamma) .
$$

Since $f$ is of bounded variation on $[a, b]$, then we have

$$
\begin{aligned}
& \sup _{t \in[a, b]}\left|f(t)-\frac{f(a)+f(b)}{2}\right| \\
& =\sup _{t \in[a, b]}\left|\frac{f(t)-f(a)+f(t)-f(b)}{2}\right| \\
& \leq \frac{1}{2} \sup _{t \in[a, b]}[|f(t)-f(a)|+|f(t)-f(b)|] \leq \frac{1}{2} \bigvee_{a}^{b}(f),
\end{aligned}
$$

for all $t \in[a, b]$. Finally, combining the inequalities (2.7)-(2.9), we obtain the required result (2.6).

Assume that (2.6) holds with a constant $C>0$,i.e.,

$$
|\mathcal{T}(f, g ; u)| \leq C(\Gamma-\gamma) \bigvee_{a}^{b}(f)
$$

Consider the functions $u(t)=t$ and $f(t)=g(t)=\operatorname{sgn}\left(t-\frac{a+b}{2}\right), t \in[a, b]$. Then $\bigvee_{a}^{b}(f)=2, \Gamma-\gamma=2$ and

$$
\frac{1}{b-a} \int_{a}^{b} f(t) g(t) d t=1, \int_{a}^{b} f(t) d t=\int_{a}^{b} g(t) d t=0
$$

and from (2.10) we get $C \geq \frac{1}{4}$ which proves the sharpness of the constant.

We have the following result as well:

Theorem 2.4. Let $g:[a, b] \rightarrow \mathbb{C}$ be such that $g$ is of bounded variation on $[a, b]$ and $u$ be a monotonic nondecreasing functions on $[a, b]$, then we have

$$
|\mathcal{T}(f, g ; u)| \leq\left\{\begin{array}{l}
H_{f}(b-a)^{p} \bigvee_{a}^{b}(g), \quad \text { if } f \text { is } H_{f} \text {-p-Hölder } \\
\frac{1}{2} \bigvee_{a}^{b}(f) \bigvee_{a}^{b}(g), \quad \text { if } f \text { is of bounded variation }
\end{array}\right.
$$

where, $H_{f}>0$ and $p \in(0,1]$ are given. 
Proof. Using (1.4) we may write

$$
\begin{aligned}
& |\mathcal{T}(f, g ; u)| \\
& \leq \frac{1}{u(b)-u(a)} \int_{a}^{b}\left|f(t)-\frac{f(a)+f(b)}{2}\right| \\
& \times\left|g(t)-\frac{1}{u(b)-u(a)} \int_{a}^{b} g(s) d u(s)\right| d u(t) \\
& =\frac{1}{(u(b)-u(a))^{2}} \int_{a}^{b}\left|f(t)-\frac{f(a)+f(b)}{2}\right| \\
& \times\left|\int_{a}^{b}[g(t)-g(s)] d u(s)\right| d u(t) \\
& \leq \frac{1}{(u(b)-u(a))^{2}} \\
& \times \int_{a}^{b}\left[\left|f(t)-\frac{f(a)+f(b)}{2}\right| \int_{a}^{b}|g(t)-g(s)| d u(s)\right] d u(t) .
\end{aligned}
$$

But since $g$ is of bounded variation we then have

$$
\begin{aligned}
\int_{a}^{b}|g(t)-g(s)| d u(s) & \leq \sup _{s \in[a, b]}|g(t)-g(s)| \int_{a}^{b} d u(s) \\
& \leq \bigvee_{a}^{b}(g)[u(b)-u(a)] .
\end{aligned}
$$

Therefore, if $f$ is of $p$-Hölder type, then we have

$$
\begin{aligned}
& |\mathcal{T}(f, g ; u)| \\
& \leq \frac{1}{2} \frac{1}{u(b)-u(a)} \bigvee_{a}^{b}(g) \int_{a}^{b}[|f(t)-f(a)|+|f(t)-f(b)|] d u(t) \\
& \leq \frac{H_{f}}{2(u(b)-u(a))} \bigvee_{a}^{b}(g) \int_{a}^{b}\left[|t-a|^{p}+|t-b|^{p}\right] d u(t) \\
& =\frac{H_{f}}{2(u(b)-u(a))} \bigvee_{a}^{b}(g) \int_{a}^{b}\left[(t-a)^{p}+(b-t)^{p}\right] d u(t) .
\end{aligned}
$$

We have

$$
\int_{a}^{b}\left[(t-a)^{p}+(b-t)^{p}\right] d u(t)=\int_{a}^{b}(t-a)^{p} d u(t)+\int_{a}^{b}(b-t)^{p} d u(t) .
$$

Utilising the integration by parts formula, we have

$$
\int_{a}^{b}(t-a)^{p} d u(t)=(b-a)^{p} u(b)-p \int_{a}^{b} u(t)(t-a)^{p-1} d t
$$


and

$$
\int_{a}^{b}(b-t)^{p} d u(t)=-(b-a)^{p} u(a)+p \int_{a}^{b} u(t)(b-t)^{p-1} d t .
$$

Now, since $u$ is monotonic we have

$$
\int_{a}^{b} u(t)(t-a)^{p-1} d t \geq \frac{1}{p}(b-a)^{p} u(a)
$$

and

$$
\int_{a}^{b} u(t)(b-t)^{p-1} d t \leq \frac{1}{p}(b-a)^{p} u(b) .
$$

Thus, by (2.14), we get

$$
\begin{aligned}
\int_{a}^{b}\left[(t-a)^{p}+(b-t)^{p}\right] d u(t) & =\int_{a}^{b}(t-a)^{p} d u(t)+\int_{a}^{b}(b-t)^{p} d u(t) \\
& \leq 2(b-a)^{p}[u(b)-u(a)]
\end{aligned}
$$

which gives by (2.13), that

$$
\begin{aligned}
|\mathcal{T}(f, g ; u)| & \leq \frac{H_{f}}{2(u(b)-u(a))} \bigvee_{a}^{b}(g) \int_{a}^{b}\left[(t-a)^{p}+(b-t)^{p}\right] d u(t) \\
& \leq H_{f}(b-a)^{p} \bigvee_{a}^{b}(g)
\end{aligned}
$$

which prove the first part of inequality (2.11).

To prove the second part of (2.11), assume that $f$ is of bounded variation, then we have

$$
\begin{aligned}
& |\mathcal{T}(f, g ; u)| \\
& \leq \frac{1}{2} \frac{1}{u(b)-u(a)} \bigvee_{a}^{b}(g) \int_{a}^{b}[|f(t)-f(a)|+|f(t)-f(b)|] d u(t) \\
& \leq \frac{1}{2} \bigvee_{a}^{b}(g) \bigvee_{a}^{b}(f)
\end{aligned}
$$

and thus the theorem is proved.

Theorem 2.5. Let $g, u:[a, b] \rightarrow \mathbb{R}$ be such that $g$ is of $q-H_{g}-H$ ölder type on $[a, b]$, and $u$ be a monotonic nondecreasing functions on $[a, b]$, then we have

$$
|\mathcal{T}(f, g ; u)| \leq \begin{cases}H_{g}(b-a)^{q} \bigvee_{a}^{b}(f), & \text { if } f \text { is of bounded variation } \\ \frac{1}{2^{p}} H_{f} H_{g}(b-a)^{p+q}, & \text { if } f \text { is } H_{f} \text {-p-Hölder }\end{cases}
$$

where $H_{f}>0$ and $p \in(0,1]$ are given . 
Proof. Using (1.4), we may write

$$
\begin{aligned}
& |\mathcal{T}(f, g ; u)| \\
& \leq \frac{1}{u(b)-u(a)} \int_{a}^{b}\left|f(t)-\frac{f(a)+f(b)}{2}\right| \\
& \times\left|g(t)-\frac{1}{u(b)-u(a)} \int_{a}^{b} g(s) d u(s)\right| d u(t) \\
& =\frac{1}{(u(b)-u(a))^{2}} \int_{a}^{b}\left|f(t)-\frac{f(a)+f(b)}{2}\right| \\
& \times\left|\int_{a}^{b}[g(t)-g(s)] d u(s)\right| d u(t) \\
& \leq \frac{1}{(u(b)-u(a))^{2}} \\
& \times \int_{a}^{b}\left[\left|f(t)-\frac{f(a)+f(b)}{2}\right| \int_{a}^{b}|g(t)-g(s)| d u(s)\right] d u(t) \\
& \leq \frac{1}{(u(b)-u(a))^{2}} \sup _{t \in[a, b]}\left|f(t)-\frac{f(a)+f(b)}{2}\right| \\
& \times \int_{a}^{b}\left[\int_{a}^{b}|g(t)-g(s)| d u(s)\right] d u(t) \\
& \leq \frac{1}{2(u(b)-u(a))^{2}} \bigvee_{a}^{b}(f) \int_{a}^{b}\left[\int_{a}^{b}|g(t)-g(s)| d u(s)\right] d u(t) .
\end{aligned}
$$

Since $g$ is of $q-H_{g}$-Holder type on $[a, b]$, then we have

$$
\begin{aligned}
& |\mathcal{T}(f, g ; u)| \\
& \leq \frac{1}{2(u(b)-u(a))^{2}} \bigvee_{a}^{b}(f) \int_{a}^{b}\left[\int_{a}^{b}|g(t)-g(s)| d u(s)\right] d u(t) \\
& \leq \frac{H_{g}}{2(u(b)-u(a))^{2}} \bigvee_{a}^{b}(f) \int_{a}^{b}\left[\int_{a}^{b}|t-s|^{q} d u(s)\right] d u(t) .
\end{aligned}
$$


Now, using the Riemann-Stieltjes integral and then utilizing the monotonicity of $u$ on $[a, b]$ (twice), we get

$$
\begin{gathered}
\int_{a}^{b}\left[\int_{a}^{b}|t-s|^{q} d u(s)\right] d u(t) \\
=\int_{a}^{b}\left[\int_{a}^{t}(s-a)^{q} d u(s)+\int_{t}^{b}(b-s)^{q} d u(s)\right] d u(t) \\
=\int_{a}^{b}\left[(t-a)^{q} u(t)-q \int_{a}^{t}(s-a)^{q-1} u(s) d s\right. \\
\left.-(b-t)^{q} u(t)+q \int_{t}^{b}(b-s)^{q-1} u(s) d s\right] d u(t) \\
\leq \int_{a}^{b}\left[(t-a)^{q} u(t)-(t-a)^{q} u(a)-(b-t)^{q} u(t)+(b-t)^{q} u(b)\right] d u(t) \\
\leq \int_{a}^{b}\left[(t-a)^{q} u(b)-(t-a)^{q} u(a)-(b-t)^{q} u(a)+(b-t)^{q} u(b)\right] d u(t) \\
=[u(b)-u(a)] \int_{a}^{b}\left[(t-a)^{q}+(b-t)^{q}\right] d u(t) \\
\leq 2(b-a)^{q}[u(b)-u(a)]^{2}, \quad b y(2.15) .
\end{gathered}
$$

which proves the first part of (2.16).

To prove the second part of (2.16), assume that $f$ is of $p-H_{f}-$ Hölder type, then we have

$$
\begin{aligned}
& |\mathcal{T}(f, g ; u)| \\
& \leq \frac{1}{(u(b)-u(a))^{2}} \sup _{t \in[a, b]}\left|f(t)-\frac{f(a)+f(b)}{2}\right| \\
& \times \int_{a}^{b}\left[\int_{a}^{b}|g(t)-g(s)| d u(s)\right] d u(t) \\
& \leq \frac{H_{g}}{2(u(b)-u(a))^{2}} H_{f}\left(\frac{b-a}{2}\right)^{p} \int_{a}^{b}\left[\int_{a}^{b}|t-s|^{q} d u(s)\right] d u(t) \\
& =\frac{H_{g}}{2(u(b)-u(a))^{2}} H_{f}\left(\frac{b-a}{2}\right)^{p} 2(b-a)^{q}(u(b)-u(a))^{2} \\
& =\frac{1}{2^{p}} H_{g} H_{f}(b-a)^{p+q}
\end{aligned}
$$

which proves the second part of (2.16), and thus the proof is established.

\section{Generalizations of some ReCEnt Results}

In this section we generalize some Grüss type inequalities for Lebesgue-Stieltjes integral.

In the recent work [7], Dragomir has proved the following inequality: 
Theorem 3.1. Let $f:[a, b] \rightarrow \mathbb{C}$ be of bounded variation on $[a, b]$ and $g:[a, b] \rightarrow$ $\mathbb{C}$ a Lebesgue integrable function on $[a, b]$, then

$$
|\mathcal{T}(f, g)| \leq \frac{1}{2} \bigvee_{a}^{b}(f) \frac{1}{b-a} \int_{a}^{b}\left|g(t)-\frac{1}{b-a} \int_{a}^{b} g(s) d s\right| d t,
$$

where, $\bigvee_{a}^{b}(f)$ denotes the total variation of $f$ on the interval $[a, b]$. The constant $\frac{1}{2}$ is best possible in (3.1).

Another result when both functions are of bounded variation, was considered in the same paper [7], as follows:

Theorem 3.2. If $f, g:[a, b] \rightarrow \mathbb{C}$ are of bounded variation on $[a, b]$, then

$$
|\mathcal{T}(f, g)| \leq \frac{1}{4} \bigvee_{a}^{b}(f) \bigvee_{a}^{b}(g)
$$

The constant $\frac{1}{4}$ is best possible in (3.2).

The following result holds:

Theorem 3.3. Let $f:[a, b] \rightarrow \mathbb{C}$ be of bounded variation on $[a, b]$ and $g:[a, b] \rightarrow$ $\mathbb{C}$ be such that $g$ is a Lebesgue-Stieltjes integrable with respect to a monotonic nondecreasing function $u$ on $[a, b]$, then

$$
\begin{aligned}
& |\mathcal{T}(f, g ; u)| \\
& \leq \frac{1}{2} \bigvee_{a}^{b}(f) \frac{1}{u(b)-u(a)} \int_{a}^{b}\left|g(t)-\frac{1}{u(b)-u(a)} \int_{a}^{b} g(s) d u(s)\right| d u(t) .
\end{aligned}
$$

The constant $\frac{1}{2}$ is best possible.

Proof. Using (1.4) and utilizing the triangle inequality, we get

$$
\begin{aligned}
& |\mathcal{T}(f, g ; u)| \\
& \leq \frac{1}{u(b)-u(a)} \int_{a}^{b}\left|f(t)-\frac{f(a)+f(b)}{2}\right| \\
& \times\left|g(t)-\frac{1}{u(b)-u(a)} \int_{a}^{b} g(s) d u(s)\right| d u(t) \\
& \leq \frac{1}{u(b)-u(a)} \sup _{t \in[a, b]}\left|f(t)-\frac{f(a)+f(b)}{2}\right| \\
& \times \int_{a}^{b}\left|g(t)-\frac{1}{u(b)-u(a)} \int_{a}^{b} g(s) d u(s)\right| d u(t) \\
& \leq \frac{1}{2} \bigvee_{a}^{b}(f) \frac{1}{u(b)-u(a)} \int_{a}^{b}\left|g(t)-\frac{1}{u(b)-u(a)} \int_{a}^{b} g(s) d u(s)\right| d u(t) .
\end{aligned}
$$

Since $f$ is of bounded variation on $[a, b]$, then we have that

$$
|f(t)-f(a)|+|f(t)-f(b)| \leq \bigvee_{a}^{b}(f)
$$


for all $t \in[a, b]$.

The sharpness of (3.3) follows immediately by taking $u(t)=t$ and $f(t)=$ $\operatorname{sgn}\left(t-\frac{a+b}{2}\right)$ and $g(t)=\left(t-\frac{a+b}{2}\right)$. For the details see [7].

The variance of the function $f:[a, b] \rightarrow \mathbb{C}$ which is square integrable on $[a, b]$ by $D(f)$ and is defined as [7]:

$$
\begin{aligned}
D(f) & :=[T(f, \bar{f})]^{1 / 2} \\
& =\left[\frac{1}{b-a} \int_{a}^{b}|f(t)|^{2} d t-\left|\frac{1}{b-a} \int_{a}^{b} f(t) d t\right|^{2}\right]^{1 / 2},
\end{aligned}
$$

where $\bar{f}$ denotes the complex conjugate function of $f$.

A generalization of $D(f)$ in terms of Lebesgue-Stieltjes integral may be considered by defining the variance of the function $f:[a, b] \rightarrow \mathbb{C}$ which is square integrable with respect to a monotonic nondecreasing function $u:[a, b] \rightarrow \mathbb{C}$ on $[a, b]$ by $D(f ; u)$ and is defined as:

$$
\begin{aligned}
D(f ; u) & :=[T(f, \bar{f} ; u)]^{1 / 2} \\
& =\left[\frac{1}{u(b)-u(a)} \int_{a}^{b}|f(t)|^{2} d u(t)-\left|\frac{1}{u(b)-u(a)} \int_{a}^{b} f(t) d u(t)\right|^{2}\right]^{1 / 2} .
\end{aligned}
$$

Corollary 3.4. Let $f:[a, b] \rightarrow \mathbb{C}$ be a function of bounded variation on $[a, b]$, then

$$
D(f ; u) \leq \frac{1}{2} \bigvee_{a}^{b}(f) .
$$

Proof. Applying Theorem 3.3 for $g=\bar{f}$ we get

$$
\begin{aligned}
& D^{2}(f ; u) \\
& \leq \frac{1}{2} \bigvee_{a}^{b}(f) \frac{1}{u(b)-u(a)} \int_{a}^{b}\left|f(t)-\frac{1}{u(b)-u(a)} \int_{a}^{b} f(s) d u(s)\right| d u(t) .
\end{aligned}
$$

By the Cauchy-Bunyakovsky-Schwarz integral inequality we have

$$
\frac{1}{u(b)-u(a)} \int_{a}^{b}\left|f(t)-\frac{1}{u(b)-u(a)} \int_{a}^{b} f(s) d u(s)\right| d u(t) \leq D(f ; u) \text {. }
$$

Making use of (3.7) and (3.8) we get (3.6).

The sharpness follows by taking $u(t)=t$ and $f(t)=\operatorname{sgn}\left(t-\frac{a+b}{2}\right)$. We omit the details.

Remark 3.5. If in Theorems 2.1-2.5, we set $g=\bar{f}$, then several bounds for $D(f ; u)$ under various assumptions for the functions involved may be deduced. The details are omitted.

In the following we obtain another result when both functions $f, g$ are of bounded variation, which therefore generalizes Theorem 3.2 and refines the second inequality of (2.11): 
Theorem 3.6. Let $f, g:[a, b] \rightarrow \mathbb{C}$ be two functions of bounded variation on $[a, b]$, then

$$
|\mathcal{T}(f, g ; u)| \leq \frac{1}{4} \bigvee_{a}^{b}(f) \bigvee_{a}^{b}(g)
$$

The constant $\frac{1}{4}$ is the best possible.

Proof. On making use of Theorem 3.3 and Corollary 3.4 we have

$$
\begin{aligned}
& |\mathcal{T}(f, g ; u)| \\
& \leq \frac{1}{2} \bigvee_{a}^{b}(f) \frac{1}{u(b)-u(a)} \int_{a}^{b}\left|g(t)-\frac{1}{u(b)-u(a)} \int_{a}^{b} g(s) d u(s)\right| d u(t) \\
& \leq \frac{1}{2} \bigvee_{a}^{b}(f) D(g ; u) \leq \frac{1}{4} \bigvee_{a}^{b}(f) \bigvee_{a}^{b}(g) .
\end{aligned}
$$

The case of equality is obtained in (3.9) for $f(t)=g(t)=\operatorname{sgn}\left(t-\frac{a+b}{2}\right), t \in$ $[a, b]$.

Remark 3.7. By reconsidering the functions in Theorems 2.1-2.5 to be complex valued functions and according to Remark 3.5, one may obtain several bounds for $\mathcal{T}(f, g ; u)$ using $D(f ; u)$. The details are left to the interested readers.

\section{Applications FOR SELFAdjoint operators}

We denote by $\mathcal{B}(H)$ the Banach algebra of all bounded linear operators on a complex Hilbert space $(H ;\langle\cdot, \cdot\rangle)$. Let $A \in \mathcal{B}(H)$ be selfadjoint and let $\varphi_{\lambda}$ be defined for all $\lambda \in \mathbb{R}$ as follows

$$
\varphi_{\lambda}(s):=\left\{\begin{array}{l}
1, \text { for }-\infty<s \leq \lambda \\
0, \text { for } \lambda<s<+\infty
\end{array}\right.
$$

Then for every $\lambda \in \mathbb{R}$ the operator

$$
E_{\lambda}:=\varphi_{\lambda}(A)
$$

is a projection which reduces $A$.

The properties of these projections are collected in the following fundamental result concerning the spectral representation of bounded selfadjoint operators in Hilbert spaces, see for instance [22, p. 256]:

Theorem 4.1 (Spectral Representation Theorem). Let $A$ be a bonded selfadjoint operator on the Hilbert space $H$ and let $m=\min \{\lambda \mid \lambda \in S p(A)\}=: \min S p(A)$ and $M=\max \{\lambda \mid \lambda \in S p(A)\}=: \max S p(A)$. Then there exists a family of projections $\left\{E_{\lambda}\right\}_{\lambda \in \mathbb{R}}$, called the spectral family of $A$, with the following properties

a) $E_{\lambda} \leq E_{\lambda^{\prime}}$ for $\lambda \leq \lambda^{\prime}$

b) $E_{m-0}=0, E_{M}=I$ and $E_{\lambda+0}=E_{\lambda}$ for all $\lambda \in \mathbb{R}$; 
c) We have the representation

$$
A=\int_{m-0}^{M} \lambda d E_{\lambda}
$$

More generally, for every continuous complex-valued function $\varphi$ defined on $\mathbb{R}$ and for every $\varepsilon>0$ there exists a $\delta>0$ such that

$$
\left\|\varphi(A)-\sum_{k=1}^{n} \varphi\left(\lambda_{k}^{\prime}\right)\left(E_{\lambda_{k}}-E_{\lambda_{k-1}}\right)\right\| \leq \varepsilon
$$

whenever

this means that

$$
\left\{\begin{array}{l}
\lambda_{0}<m=\lambda_{1}<\cdots<\lambda_{n-1}<\lambda_{n}=M, \\
\lambda_{k}-\lambda_{k-1} \leq \delta \text { for } 1 \leq k \leq n, \\
\lambda_{k}^{\prime} \in\left[\lambda_{k-1}, \lambda_{k}\right] \text { for } 1 \leq k \leq n
\end{array}\right.
$$

$$
\varphi(A)=\int_{m-0}^{M} \varphi(\lambda) d E_{\lambda}
$$

where the integral is of Riemann-Stieltjes type.

Corollary 4.2. With the assumptions of Theorem 4.1 for $A, E_{\lambda}$ and $\varphi$ we have the representations

$$
\varphi(A) x=\int_{m-0}^{M} \varphi(\lambda) d E_{\lambda} x \quad \text { for all } x \in H
$$

and

$$
\langle\varphi(A) x, y\rangle=\int_{m-0}^{M} \varphi(\lambda) d\left\langle E_{\lambda} x, y\right\rangle \quad \text { for all } x, y \in H .
$$

In particular,

$$
\langle\varphi(A) x, x\rangle=\int_{m-0}^{M} \varphi(\lambda) d\left\langle E_{\lambda} x, x\right\rangle \quad \text { for all } x \in H .
$$

Moreover, we have the equality

$$
\|\varphi(A) x\|^{2}=\int_{m-0}^{M}|\varphi(\lambda)|^{2} d\left\|E_{\lambda} x\right\|^{2} \quad \text { for all } x \in H .
$$

We observe that the function $u(\lambda):=\left\langle E_{\lambda} x, x\right\rangle$ is monotonic nondecreasing and right continuous on $[m, M]$.

Theorem 4.3. Let $A$ be a bonded selfadjoint operator on the Hilbert space $H$ and let $m=\min \{\lambda \mid \lambda \in S p(A)\}=: \min S p(A)$ and $M=\max \{\lambda \mid \lambda \in S p(A)\}$ $=: \max S p(A)$.

(i) If $f:[m, M] \rightarrow \mathbb{C}$ is a $p-H_{f}-$ Hölder continuous function on $[m, M]$, where $p \in(0,1]$ and $H_{f}>0$ are given and $g:[m, M] \rightarrow \mathbb{R}$ is continuous and $\gamma=\min _{t \in[m, M]} g(t), \Gamma=\max _{t \in[m, M]} g(t)$, then

$$
|C(f, g ; A ; x)| \leq \frac{1}{2^{p+1}} H_{f}(\Gamma-\gamma)(M-m)^{p},
$$


where $C(f, g ; A ; x)$ denotes the following Čebyšev functional

$$
C(f, g ; A ; x):=\langle f(A) g(A) x, x\rangle-\langle f(A) x, x\rangle \cdot\langle g(A) x, x\rangle
$$

and $x \in H$ with $\|x\|=1$.

(ii) If $f:[m, M] \rightarrow \mathbb{C}$ is continuous and of bounded variation on $[m, M]$ while $g$ is as in (i), then

$$
|C(f, g ; A ; x)| \leq \frac{1}{4}(\Gamma-\gamma) \bigvee_{m}^{M}(f)
$$

for any $x \in H$ with $\|x\|=1$.

(iii) If the function $f$ is as in (i) and $g:[m, M] \rightarrow \mathbb{C}$ is continuous and of bounded variation on $[m, M]$, then

$$
|C(f, g ; A ; x)| \leq H_{f} \bigvee_{m}^{M}(g)(M-m)^{p}
$$

for any $x \in H$ with $\|x\|=1$.

(iv) If $f$ is of bounded variation and $g$ is continuous, then

$$
|C(f, g ; A ; x)| \leq \frac{1}{2}\left\langle\left|g(A)-\langle g(A) x, x\rangle 1_{H}\right| x, x\right\rangle \bigvee_{m}^{M}(f)
$$

for any $x \in H$ with $\|x\|=1$. Moreover, if $g$ is also of bounded variation, then we have the simpler bound

$$
\begin{aligned}
|C(f, g ; A ; x)| & \leq \frac{1}{2}\left\langle\left|g(A)-\langle g(A) x, x\rangle 1_{H}\right| x, x\right\rangle \bigvee_{m}^{M}(f) \\
& \leq \frac{1}{4} \bigvee_{m}^{M}(f) \bigvee_{m}^{M}(g)
\end{aligned}
$$

for any $x \in H$ with $\|x\|=1$.

Proof. (i) Fix $x \in H$ with $\|x\|=1$. Let $s>0$ and extend by continuity the functions $f$ and $g$ to the interval $[m-s, M]$ by preserving their properties from $[m, M]$. Consider also the monotonic function $u(\lambda):=\left\langle E_{\lambda} x, x\right\rangle$ which is monotonic nondecreasing and right continuous on $[m-s, M]$.

Now, writing the inequality (2.1) for these functions we have

$$
\begin{aligned}
& \mid \frac{1}{u(M)-u(m-s)} \int_{m-s}^{M} f(\lambda) g(\lambda) d u(\lambda) \\
& -\frac{1}{u(M)-u(m-s)} \int_{m-s}^{M} f(\lambda) d u(\lambda) \\
& \times \frac{1}{u(M)-u(m-s)} \int_{m-s}^{M} g(\lambda) d u(\lambda) \mid \\
& \leq \frac{1}{2^{p+1}} H_{f}(\Gamma-\gamma)(M-m+s)^{p} .
\end{aligned}
$$


Now, by letting $s \rightarrow 0+$ and utilizing the representation (4.8) and the fact that $u(M)=1, u(m-0)=0$ we get

$$
|\langle f(A) g(A) x, x\rangle-\langle f(A) x, x\rangle\langle g(A) x, x\rangle| \leq \frac{1}{2^{p+1}} H_{f}(\Gamma-\gamma)(M-m)^{p}
$$

for $x \in H$ with $\|x\|=1$.

This proves the inequality (4.10).

The statement (ii) follows from (2.6), (iii) follows from (2.11), (iv) follows from (3.3) and (3.10) by employing a similar argument.

The details are left to the interested reader.

The above results may provide some interesting inequalities for fundamental functions of operators such as the power and logarithmic functions.

If we consider the function $f:[0, \infty) \rightarrow \mathbb{R}, f(t)=t^{p}$ with $p \in(0,1)$ then we observe that this functions is $p$-Hölder continuous with the constant $H_{f}=1$ on any subinterval from $[0, \infty)$. So, if $A$ is a positive operator on the Hilbert space $H$ with the spectrum $S p(A) \subset[m, M] \subset[0, \infty)$ then from (4.10) we have the inequality

$$
\left|\left\langle A^{p} g(A) x, x\right\rangle-\left\langle A^{p} x, x\right\rangle\langle g(A) x, x\rangle\right| \leq \frac{1}{2^{p+1}}(\Gamma-\gamma)(M-m)^{p}
$$

with $x \in H$ with $\|x\|=1$ and for any function $g:[m, M] \rightarrow \mathbb{R}$ that is continuous and $\gamma=\min _{t \in[m, M]} g(t), \Gamma=\max _{t \in[m, M]} g(t)$.

Now, if in (4.17) we choose $g(t)=t^{q}$ with $q>0$, then we get the inequality

$$
\left|\left\langle A^{p+q} x, x\right\rangle-\left\langle A^{p} x, x\right\rangle\left\langle A^{q} x, x\right\rangle\right| \leq \frac{1}{2^{p+1}}\left(M^{q}-m^{q}\right)(M-m)^{p}
$$

for any $x \in H$ with $\|x\|=1$.

If $m>0$, then by choosing $g(t)=\ln t$ we get the logarithmic inequality

$$
\left|\left\langle A^{p} \ln A x, x\right\rangle-\left\langle A^{p} x, x\right\rangle\langle\ln A x, x\rangle\right| \leq \frac{1}{2^{p+1}} \ln \left(\frac{M}{m}\right)(M-m)^{p}
$$

for any $x \in H$ with $\|x\|=1$.

Now, if we take the function $g(t)=t^{q}$ with $q>0$ and if $A$ is a positive operator on the Hilbert space $H$ with the spectrum $S p(A) \subset[m, M] \subset[0, \infty)$, then we get from (4.11)

$$
\left|\left\langle f(A) A^{q} x, x\right\rangle-\langle f(A) x, x\rangle\left\langle A^{q} x, x\right\rangle\right| \leq \frac{1}{4}\left(M^{q}-m^{q}\right) \bigvee_{m}^{M}(f)
$$

for any $x \in H$ with $\|x\|=1$, where $f:[m, M] \rightarrow \mathbb{C}$ is continuous and of bounded variation on $[m, M]$.

If we take in (4.20) $f(t)=t^{p}$ with $p>0$, then we get

$$
\left|\left\langle A^{p+q} x, x\right\rangle-\left\langle A^{p} x, x\right\rangle\left\langle A^{q} x, x\right\rangle\right| \leq \frac{1}{4}\left(M^{p}-m^{p}\right)\left(M^{q}-m^{q}\right)
$$

for any $x \in H$ with $\|x\|=1$. 
If $m>0$, then by choosing $g(t)=\ln t$ we get the logarithmic inequality

$$
\left|\left\langle A^{q} \ln A x, x\right\rangle-\langle\ln A x, x\rangle\left\langle A^{q} x, x\right\rangle\right| \leq \frac{1}{4} \ln \left(\frac{M}{m}\right)\left(M^{q}-m^{q}\right)
$$

for any $x \in H$ with $\|x\|=1$.

Remark 4.4. The interested reader in operator inequalities may be able to find other recent results providing various bounds for the Čebyšev functional $C(f, g ; A ; x)$ in the papers [12]-[18] and the monograph [19].

\section{REFERENCES}

1. M.W. Alomari and S.S. Dragomir, New Grüss type inequalities for the Stieltjes integral with applications, submitted. Avalibale at [http://ajmaa.org/RGMIA/papers/v15/v15a.pdf].

2. G.A. Anastassiou, Grüss type inequalities for the Stieltjes integral, Nonlinear Funct. Anal. Appl. 12 (2007), no. 4, 583-593.

3. G.A. Anastassiou, Chebyshev-Grüss type and comparison of integral means inequalities for the Stieltjes integral, Panamer. Math. J. 17 (2007), no. 3, 91-109.

4. P. Cerone and S.S. Dragomir, New bounds for the Čebyšev functional, Appl. Math. Lett. 18 (2005) 603-611.

5. P. Cerone and S.S. Dragomir, A refinement of the Grüss inequality and applications, Tamkang J. Math. 38 (2007), no. 1, 37-49.

6. S.S. Dragomir, Inequalities of Grüss type for the Stieltjes integral and applications, Kragujevac J. Math. 26 (2004) 89-112.

7. S.S. Dragomir, New Grüss' type inequalities for functions of bounded variation and applications, Appl. Math. Lett. 25 (2012), no. 10, p. 1475-1479.

8. S.S. Dragomir, New estimates of the Čebyšev functional for Stieltjes integrals and applications, J. Korean Math. Soc. 41 (2004), no. 2, 249-264.

9. S.S. Dragomir, Sharp bounds of Čebyšev functional for Stieltjes integrals and applications, Bull. Austral. Math. Soc. 67 (2003), no. 2, 257-266.

10. S.S. Dragomir, Some integral inequality of Grüss type, Indian J. Pure Appl. Math. 31 (2000), no. 4, 397-415.

11. S.S. Dragomir, Grüss type integral inequality for mappings of $r$-Hölder type and applications for trapezoid formula, Tamkang J. Math. 31 (2000), no. 1, 43-47.

12. S.S. Dragomir, Čebyšev's type inequalities for functions of selfadjoint operators in Hilbert spaces, Linear Multilinear Algebra 58 (2010), no. 7, 805-814.

13. S.S. Dragomir, Grüss' type inequalities for functions of selfadjoint operators in Hilbert spaces, Preprint RGMIA Res. Rep. Coll. 11(e) (2008), Art. 11.

14. S.S. Dragomir, Some new Grüss' type inequalities for functions of selfadjoint operators in Hilbert spaces, Sarajevo J. Math. 18 (2010), 89-107.

15. S.S. Dragomir, Inequalities for the Čebyšev functional of two functions of selfadjoint operators in Hilbert spaces, Aust. J. Math. Anal. Appl. 6 (2009), No. 1, Art 7.

16. S.S. Dragomir, Some inequalities for the Čebyšev functional of two functions of selfadjoint operators in Hilbert spaces, RGMIA Res. Rep. Coll. 11(e) (2008), Art. 8.

17. S.S. Dragomir, Quasi Grüss' type inequalities for continuous functions of selfadjoint operators in Hilbert spaces, Preprint RGMIA Res. Rep. Coll. 13 (2010), Sup. Art. 12.

18. S.S. Dragomir, Grüss' type inequalities for some classes of continuous functions of selfadjoint operators in Hilbert spaces, Preprint RGMIA Res. Rep. Coll. 13 (2010), no. 2, Art. 15.

19. S.S. Dragomir, Operator Inequalities of the Jensen, Čebyšev and Grüss Type. Springer Briefs in Mathematics. Springer, New York, 2012.

20. S.S. Dragomir and I. Fedotov, An inequality of Grüss type for Riemann-Stieltjes integral and applications for special means, Tamkang J. Math. 29 (1998), no. 4, 287-292. 
21. G. Grüss, Über das maximum des absoluten Betrages von $\frac{1}{b-a} \int_{a}^{b} f(x) g(x) d x-$ $\frac{1}{(b-a)^{2}} \int_{a}^{b} f(x) d x \cdot \int_{a}^{b} g(x) d x$, Math. Z. 39 (1935), 215-226.

22. G. Helmberg, Introduction to Spectral Theory in Hilbert Space, John Wiley \& Sons, Inc. -New York, 1969.

23. Z. Liu, Refinement of an inequality of Grüss type for Riemann-Stieltjes integral, Soochow J. Math. 30 (2004), no. 4, 483-489.

${ }^{1}$ Department of Mathematics, Faculty of Science, Jerash University, 26150 JERASH, JORDAN.

E-mail address: mwomath@gmail.com

2 Mathematics, School of Engineering \& Science, Victoria University, PO Box 14428, Melbourne City, MC 8001, Australia;

School of Computational and Applied Mathematics, University of the Witwatersrand, Johannesburg, Private Bag 3, Wits, 2050, South Africa.

E-mail address: sever.dragomir@vu.edu.au 